Article

\title{
Reducing a Class of Two-Dimensional Integrals to One-Dimension with an Application to Gaussian Transforms
}

\author{
Jack C. Straton
}

Department of Physics, Portland State University, Portland, OR 97207-0751, USA; straton@pdx.edu

Received: 29 July 2020; Accepted: 25 August 2020; Published: 2 September 2020

\begin{abstract}
Quantum theory is awash in multidimensional integrals that contain exponentials in the integration variables, their inverses, and inverse polynomials of those variables. The present paper introduces a means to reduce pairs of such integrals to one dimension when the integrand contains powers multiplied by an arbitrary function of $x y /(x+y)$ multiplying various combinations of exponentials. In some cases these exponentials arise directly from transition-amplitudes involving products of plane waves, hydrogenic wave functions, and Yukawa and/or Coulomb potentials. In other cases these exponentials arise from Gaussian transforms of such functions.
\end{abstract}

Keywords: antihydrogen; radiative attachment; photodetachment; antihydrogen ion; analytical; hydrogen ion

\section{Introduction}

Prudnikov, Brychkov, and Marichev [1] provided a means to reduce a class of two-dimensional integrals involving exponentials and a general function of the integration variables in the specific configuration $f\left(\frac{x y}{x+y}\right)$ to a one-dimensional integral involving that same function of the new integration variable $f(t)$ :

$$
\int_{0}^{\infty} \int_{0}^{\infty} \frac{1}{\sqrt{x+y}} f\left(\frac{x y}{x+y}\right) e^{-p x-q y} d x d y=\frac{\sqrt{\pi}}{\sqrt{p q(p+q)}} \int_{0}^{\infty} e^{-(\sqrt{p}+\sqrt{q})^{2} t} f(t) d t
$$

As may be seen with comparison to the specific cases, such as entry number 3.1.3.5 on the same page, the coefficient is in error. This reduction should read

$$
\int_{0}^{\infty} \int_{0}^{\infty} \frac{1}{\sqrt{x+y}} f\left(\frac{x y}{x+y}\right) e^{-p x-q y} d x d y=\frac{\sqrt{\pi}(\sqrt{p}+\sqrt{q})}{\sqrt{p q}} \int_{0}^{\infty} e^{-(\sqrt{p}+\sqrt{q})^{2} t} f(t) d t
$$

Numerical integration of both forms, with $f(t)=t^{3 / 2}, p=3$, and $q=5$, gives 0.0024537304. In the present paper we extend this reduction technique to a class of integrals that arise in Gaussian transforms of atomic, molecular, and optical transition amplitudes [2,3] in which the above exponentials on the left-hand side may contain more complicated forms, $e^{-\frac{a}{x}-\frac{b}{y}-c x y /(x+y)-h y /(x+y)-j /(x+y)-p x-q y}$, and one may also have positive and negative powers of $x$ and $y$. The calculation of expectation operators in percolation theory [4] may also benefit from the present technique.

We will begin with a more general form

$$
\begin{aligned}
R_{2}(n, m, v, a, b, c, h, j, p, q) & =\int_{0}^{\infty} \int_{0}^{\infty} \frac{1}{x^{n / 2} y^{m / 2}(x+y)^{v / 2}} f\left(\frac{x y}{x+y}\right) \\
& \times e^{-\frac{a}{x}-\frac{b}{y}-c x y /(x+y)-h y /(x+y)-j /(x+y)-p x-q y} d x d y
\end{aligned}
$$


The reduction is facilitated by a simultaneous change of variables to

$$
\begin{gathered}
s=\frac{x}{\sqrt{x+y}} \quad, \quad t=\frac{s y}{\sqrt{x+y}} \\
x=s^{2}+t \equiv \phi(s, t) \quad, \quad y=\frac{t\left(s^{2}+t\right)}{s^{2}} \equiv \psi(s, t) \\
\frac{\partial \phi}{\partial s} \frac{\partial \psi}{\partial t}-\frac{\partial \psi}{\partial s} \frac{\partial \phi}{\partial t}=\frac{2\left(s^{2}+t\right)^{2}}{s^{3}}
\end{gathered}
$$

where the last line is the Jacobian determinant of the transformation. The $s$ and $t$ integration limits are over $[0, \infty]$. Then

$$
\begin{aligned}
R_{2}(n, m, v, a, b, c, h, j, p, q) & =2 \int_{0}^{\infty} f(t) t^{-m / 2} e^{-c t-q t} d t \int_{0}^{\infty} s^{m+v-3}\left(s^{2}+t\right)^{-\frac{m}{2}-\frac{n}{2}-v+2} \\
& \times \exp \left(-\frac{a}{s^{2}+t}-\frac{b s^{2}}{t\left(s^{2}+t\right)}-\frac{h t}{s^{2}+t}-\frac{j s^{2}}{\left(s^{2}+t\right)^{2}}-p\left(s^{2}+t\right)-\frac{q t^{2}}{s^{2}}\right) d s
\end{aligned}
$$

\section{Set of Integral Reductions for Positive Powers in the Exponentials}

If we set $a=b=c=h=j=0$, complete the square in the exponential and change variables in the $s$ integral

$$
J_{2}(n, m, v, p, q)=\int_{0}^{\infty} s^{m+v-3}\left(s^{2}+t\right)^{-\frac{m}{2}-\frac{n}{2}-v+2} \exp \left(-p s^{2}-\frac{q t^{2}}{s^{2}}\right) d s
$$

to

$$
\begin{aligned}
u & =\sqrt{p} s-\frac{\sqrt{q} t}{s}, s=\frac{\sqrt{4 \sqrt{p} \sqrt{q} t+u^{2}}+u}{2 \sqrt{p}} \\
d s & =\frac{\left(\sqrt{4 \sqrt{p} \sqrt{q} t+u^{2}}+u\right)^{2}}{\sqrt{p}\left(\sqrt{4 \sqrt{p} \sqrt{q} t+u^{2}}+u\right)^{2}+4 p \sqrt{q} t} d u
\end{aligned}
$$

then

$$
\begin{aligned}
J_{2}(n, m, v, p, q) & =e^{-2 \sqrt{p} \sqrt{q} t} \int_{-\infty}^{\infty} e^{-u^{2}} \\
& \times \frac{2^{-m-v+3} p^{\frac{1}{2}(-m-v+3)}\left(\frac{\left(\sqrt{4 \sqrt{p} \sqrt{q} t+u^{2}}+u\right)^{2}}{4 p}+t\right)^{-\frac{m}{2}-\frac{n}{2}-v+2}}{\left(\sqrt{p}\left(\sqrt{4 \sqrt{p} \sqrt{q} t+u^{2}}+u\right)^{2}+4 p \sqrt{q} t\right)\left(\sqrt{4 \sqrt{p} \sqrt{q} t+u^{2}}+u\right)^{1-m-v}} d u
\end{aligned}
$$

2.1. Integrals with $m+v=1$

If we set $\{n=0, m=0, v=1\}$, the second factor in the denominator of (11) goes to unity, allowing us to do the integral [5], and we recover the corrected integral (2) from Prudnikov, Brychkov, and Marichev [1]:

$$
\begin{aligned}
R_{2}(00,1,0,0,0,0,0, p, q) & =\int_{0}^{\infty} \int_{0}^{\infty} \frac{1}{\sqrt{x+y}} f\left(\frac{x y}{x+y}\right) e^{-p x-q y} d x d y \\
& =\frac{\sqrt{\pi}(\sqrt{p}+\sqrt{q})}{\sqrt{p} \sqrt{q}} \int_{0}^{\infty} f(t) e^{-(\sqrt{p}+\sqrt{q})^{2} t} d t
\end{aligned}
$$


A second form may be obtained by setting

$$
f\left(\frac{x y}{x+y}\right) \equiv\left(\frac{x y}{x+y}\right)^{-1 / 2} g\left(\frac{x y}{x+y}\right)
$$

in the first form, giving

$$
\begin{aligned}
R_{2}(1,1,0,0,0,0,0,0, p, q) & =\int_{0}^{\infty} \int_{0}^{\infty} \frac{1}{x^{1 / 2} y^{1 / 2}} g\left(\frac{x y}{x+y}\right) e^{-p x-q y} d x d y \\
& =\frac{\sqrt{\pi}(\sqrt{p}+\sqrt{q})}{\sqrt{p} \sqrt{q}} \int_{0}^{\infty} t^{-1 / 2} g(t) e^{-(\sqrt{p}+\sqrt{q})^{2} t} d t
\end{aligned}
$$

Numerical integration of both forms, with $g(t)=t^{3 / 2}$, gives $R_{2}(1,1,0,0,0,0,0,0,0,0)=0.007324455$. Additional applications of (13) will give larger powers of $x$ and $y$ in the denominator with powers of $x+y$ in the numerator. Applications of the inverse of (13) will give powers of $x$ and $y$ in the numerator with larger powers of $x+y$ in the denominator.

One may also do the integral if we create even powers for the last factor in the numerator of (11) and expand by, for instance, setting $\{n=-1, m=1, v=0\}$, which gives dissimilar powers of $x$ and $y[6]$,

$$
\begin{aligned}
R_{2}(-1,1,0,0,0,0,0,0, p, q) & =\int_{0}^{\infty} \int_{0}^{\infty} \frac{x^{1 / 2}}{y^{1 / 2}} f\left(\frac{x y}{x+y}\right) e^{-p x-q y} d x d y \\
& =\frac{\sqrt{\pi}}{2 p^{3 / 2} \sqrt{q}} \int_{0}^{\infty} f(t) e^{-(\sqrt{p}+\sqrt{q})^{2} t}\left(2 t \sqrt{p}(\sqrt{p}+\sqrt{q})^{2}+\sqrt{q}\right) d t
\end{aligned}
$$

Numerical integration of both forms, with $f(t)=t^{3 / 2}$, gives $R_{2}(-1,1,0,0,0,0,0,0,3,5)=0.00281927$. The transformation (13) can be applied to this and all subsequent integrals.

\subsection{Integrals with $n+m+2 v=4$}

We obtain a new set of integral reductions by finding values of $n, m$, and $v$ that set the last factor in the numerator of (11) to unity.

2.2.1. Integrals with $v=0$

For $\{n=3, m=1, v=0\}$, and using the identity

$$
f_{1}\left(\frac{x y}{x+y}\right)=\left(\frac{x y}{x+y}\right) g\left(\frac{x y}{x+y}\right)
$$

we obtain another set of integral reductions for unlike powers of the original coordinates

$$
\begin{aligned}
R_{2}(3,1,0,0,0,0,0,0, p, q) & =R_{2}(1,-1,2,0,0,0,0,0, p, q) \\
& =\int_{0}^{\infty} \int_{0}^{\infty} \frac{\sqrt{y}}{\sqrt{x}(x+y)} g\left(\frac{x y}{x+y}\right) e^{-p x-q y} d x d y \\
& =\frac{\sqrt{\pi}}{\sqrt{q}} \int_{0}^{\infty} g(t) t^{-1 / 2} e^{-(\sqrt{p}+\sqrt{q})^{2} t} d t \quad[\Re(\sqrt{p q})>0] .
\end{aligned}
$$

Numerical integration of both forms, with $g(t)=t^{3 / 2}$, gives $R_{2}(3,1,0,0,0,0,0,0,3,5)=0.00319706$. For $\{n=5, m=-1, v=0\}$ we employ (22) to obtain 


$$
\begin{aligned}
R_{2}(5,-1,0,0,0,0,0,0, p, q) & =R_{2}(1,-5,4,0,0,0,0,0, p, q) \\
& =\int_{0}^{\infty} \int_{0}^{\infty} \frac{y^{5 / 2}}{\sqrt{x}(x+y)^{2}} g\left(\frac{x y}{x+y}\right) e^{-p x-q y} d x d y \\
& =\frac{\sqrt{\pi}}{2 q^{3 / 2}} \int_{0}^{\infty} g(t) t^{-1 / 2}(1+2 \sqrt{p q} t) e^{-(\sqrt{p}+\sqrt{q})^{2} t} d t \quad[\Re(\sqrt{p q})>0] .
\end{aligned}
$$

Numerical integration of both forms, with $g(t)=t^{3 / 2}$, gives $R_{2}(5,-1,0,0,0,0,0,0,3,5)=0.0006342548$. One may continue on in like manner.

\subsubsection{Integrals Yielding Macdonald functions}

One can also set $\{n=1, m=1, v=1\}$ to set the $\left(s^{2}+t\right)$ term of (8) to unity to obtain [7]

$$
\begin{aligned}
R_{2}(1,1,1,0,0,0,0,0, p, q) & =\int_{0}^{\infty} \int_{0}^{\infty} \frac{1}{x^{1 / 2} y^{1 / 2}(x+y)^{1 / 2}} f\left(\frac{x y}{x+y}\right) e^{-p x-q y} d x d y \\
& =2 \int_{0}^{\infty} f(t) t^{-1 / 2} e^{-p t-q t} K_{0}(2 \sqrt{p q} t) d t \quad[\Re(\sqrt{p q})>0]
\end{aligned}
$$

Numerical integration of both forms, with $f(t)=t^{3 / 2}$, gives $R_{2}(1,1,1,0,0,0,0,0,3,5)=0.01082562$. We can instead integrate [6] the $u$-form with the last factor in the numerator of (11) set to one with $\{n=1, m=1, v=1\}$. Since

$$
J_{2}(1,1,1, p, q)=J_{2}(2,2,0, p, q)
$$

we also have,

$$
\begin{aligned}
R_{2}(2,2,0,0,0,0,0,0, p, q) & =\int_{0}^{\infty} \int_{0}^{\infty} \frac{1}{x y} f\left(\frac{x y}{x+y}\right) e^{-p x-q y} d x d y \\
& =2 \int_{0}^{\infty} f(t) t^{-1} e^{-p t-q t} K_{0}(2 \sqrt{p q} t) d t \quad[\Re(\sqrt{p q})>0],
\end{aligned}
$$

but this may also be obtained from (19) by using (13). Numerical integration of both forms, with $f(t)=t^{3 / 2}$, gives $R_{2}(2,2,0,0,0,0,0,0,3,5)=0.0448319$.

We can also set the last factor in the numerator of (11) to unity with $\{n=4, m=0, v=0\}$ to obtain a set of integral reductions that have unlike powers of the original coordinates [7],

$$
\begin{aligned}
R_{2}(4,0,0,0,0,0,0,0, p, q) & =\int_{0}^{\infty} \int_{0}^{\infty} \frac{1}{x^{2}} f_{2}\left(\frac{x y}{x+y}\right) e^{-p x-q y} d x d y \\
& =2 \frac{\sqrt{p}}{\sqrt{q}} \int_{0}^{\infty} f_{3 / 2}(t) t^{-1} e^{-p t-q t} K_{1}(2 \sqrt{p q} t) d t \quad[\Re(\sqrt{p q})>0] .
\end{aligned}
$$

Numerical integration of both forms, with $f_{3 / 2}(t)=t^{3 / 2}$, gives $R_{2}(4,0,0,0,0,0,0,0,3,5)=0.1050323$. It is to be understood that each of the integral reductions in this paper is valid only for those functions $f$ that are convergent in the final integral. In the above equation we have made this restriction more explicit with the notation $f_{3 / 2}(t)$ that indicates that the integrals do not converge for terms in a powers series representation of $f$ for powers less than $3 / 2$. However, if we set

$$
f_{2}\left(\frac{x y}{x+y}\right)=\left(\frac{x y}{x+y}\right)^{2} g\left(\frac{x y}{x+y}\right)
$$

then we can rewrite 


$$
\begin{aligned}
R_{2}(4,0,0,0,0,0,0,0, p, q) & =R_{2}(0,-4,4,0,0,0,0,0, p, q) \\
& =\int_{0}^{\infty} \int_{0}^{\infty} \frac{y^{2}}{(x+y)^{2}} g\left(\frac{x y}{x+y}\right) e^{-p x-q y} d x d y \\
& =2 \frac{\sqrt{p}}{\sqrt{q}} \int_{0}^{\infty} g(t) t^{-1+2} e^{-p t-q t} K_{1}(2 \sqrt{p q} t) d t \quad[\Re(\sqrt{p q})>0],
\end{aligned}
$$

a form that converges for any function $g$ that can be expanded in a powers series (without the negative powers of a Laurent series). Numerical integration of both forms, with $g(t)=t^{0}$, gives $R_{2}(4,0,0,0,0,0,0,0,3,5)=0.0168808$. For $\{n=0, m=4, v=0\}$ we simply interchange $p \leftrightarrow q$ in the above.

Similarly, for $\{n=5, m=1, v=-1\}$ we employ (22) to obtain

$$
\begin{aligned}
R_{2}(5,1,-1,0,0,0,0,0, p, q) & =R_{2}(1,-5,4,0,0,0,0,0, p, q) \\
& =\int_{0}^{\infty} \int_{0}^{\infty} \frac{y^{3 / 2}}{\sqrt{x}(x+y)^{3 / 2}} g\left(\frac{x y}{x+y}\right) e^{-p x-q y} d x d y \\
& =2 \frac{\sqrt{p}}{\sqrt{q}} \int_{0}^{\infty} g(t) t^{1 / 2} e^{-p t-q t} K_{1}(2 \sqrt{p q} t) d t \quad[\Re(\sqrt{p q})>0] .
\end{aligned}
$$

Numerical integration of both forms, with $g(t)=t^{3 / 2}$, gives $R_{2}(4,0,0,0,0,0,0,0,3,5)=0.00128469$. One may continue on in this fashion with more extreme powers such as $\{n=7, m=-1, v=-1\}$, by using the identity

$$
f_{3}\left(\frac{x y}{x+y}\right)=\left(\frac{x y}{x+y}\right)^{3} g\left(\frac{x y}{x+y}\right)
$$

to obtain

$$
\begin{aligned}
R_{2}(7,-1,-1,0,0,0,0,0, p, q) & =R_{2}(1,-7,5,0,0,0,0,0, p, q) \\
& =\int_{0}^{\infty} \int_{0}^{\infty} \frac{y^{7 / 2}}{\sqrt{x}(x+y)^{5 / 2}} g\left(\frac{x y}{x+y}\right) e^{-p x-q y} d x d y \\
& =\frac{2 p}{q} \int_{0}^{\infty} g(t) t^{3 / 2} e^{-p t-q t} K_{2}(2 \sqrt{p q} t) d t \quad[\Re(\sqrt{p q})>0]
\end{aligned}
$$

Numerical integration of both forms, with $g(t)=t^{3 / 2}$, gives $R_{2}(7,-1,-1,0,0,0,0,0,3,5)=0.000364802$.

\subsection{Integrals with $m+v=2$}

We obtain a new set of integral reductions by finding values of $m$ and $v$ that move the second factor in the denominator of (11) to the numerator with unit power. The ratio of this term with the other term left in the denominator reduces nicely [6],

$$
\frac{\left(\sqrt{4 \sqrt{p q} t+u^{2}}+u\right)^{1}}{\left(\sqrt{p}\left(\sqrt{4 \sqrt{p q} t+u^{2}}+u\right)^{2}+4 p \sqrt{q} t\right)}=\frac{1}{\sqrt{4 \sqrt{p q} t+u^{2}}}
$$

allowing integrals of nonzero powers of the numerator of (11) to be done. We already saw the results for $\{n=1, m=1, v=1\}$ in (19). For $\{n=-1, m=1, v=1\}$ we obtain [6] 


$$
\begin{aligned}
R_{2}(-1,1,1,0,0,0,0,0, p, q)= & \int_{0}^{\infty} \int_{0}^{\infty} \frac{x^{1 / 2}}{y^{1 / 2}(x+y)^{1 / 2}} f\left(\frac{x y}{x+y}\right) e^{-p x-q y} d x d y \\
= & \frac{2}{\sqrt{p}} \int_{0}^{\infty} f(t) t^{1 / 2} e^{-p t-q t}\left(\sqrt{p} K_{0}(2 \sqrt{p q} t)+\sqrt{q} K_{1}(2 \sqrt{p q} t)\right) d t \\
& {[\Re(\sqrt{p q})>0] . }
\end{aligned}
$$

Numerical integration of both forms, with $f(t)=t^{3 / 2}$, gives $R_{2}(-1,1,1,0,0,0,0,0,3,5)=0.00324169$. For $\{n=-3, m=1, v=1\}[6]$,

$$
\begin{aligned}
R_{2}(-3,1,1,0,0,0,0,0, p, q) & =\int_{0}^{\infty} \int_{0}^{\infty} \frac{x^{3 / 2}}{y^{1 / 2}(x+y)^{1 / 2}} f\left(\frac{x y}{x+y}\right) e^{-p x-q y} d x d y \\
& =\frac{2}{p^{3 / 2}} \int_{0}^{\infty} f(t) t^{+1 / 2} e^{-p t-q t} \\
& \times\left(\sqrt{p} t(p+q) K_{0}(2 \sqrt{p q} t)+\sqrt{q}(2 p t+1) K_{1}(2 \sqrt{p q} t)\right) d t \\
& =\frac{\partial}{\partial p} R_{2}(-1,1,1,0,0,0,0,0, p, q) .
\end{aligned}
$$

Numerical integration of both forms, with $f(t)=t^{3 / 2}$, gives $R_{2}(-3,1,1,0,0,0,0,0,3,5)=0.00181426$. We have checked that this series of reductions all converge for any function $f$ that can be expanded in a powers series through $\{n=-9, m=1, v=1\}$.

\section{Integral Reductions for Inverse Powers in the Exponentials}

Instead of the positive powers in the exponential of the last section, let us take the reverse and set $p=q=h=j=0$,

$$
\begin{aligned}
R_{2}(n, m, v, a, b, c, 0,0,0,0) & =\int_{0}^{\infty} \int_{0}^{\infty} \frac{1}{x^{n / 2} y^{m / 2}(x+y)^{v / 2}} f\left(\frac{x y}{x+y}\right) \\
& \times e^{-\frac{a}{x}-\frac{b}{y}-c x y /(x+y)} d x d y . \\
& =\int_{0}^{\infty} \int_{0}^{\infty} X^{n / 2} X^{m / 2}\left(\frac{X Y}{X+Y}\right)^{v / 2} f\left(\frac{1}{X+Y}\right) \\
& \times e^{-a X-b Y-c /(X+Y)} d X d Y
\end{aligned}
$$

where we have split off $e^{-c x y /(x+y)}$ from $f\left(\frac{x y}{x+y}\right)$ to explicate how this is different from simply making the replacement $\left\{x \rightarrow \frac{1}{X}, y \rightarrow \frac{1}{Y}, a \rightarrow p, b \rightarrow q\right\}$ in Equation (3). In Equation (7) we apply a partial fraction decomposition in the exponential of

$$
-\frac{b s^{2}}{t\left(s^{2}+t\right)}=\frac{b}{s^{2}+t}-\frac{b}{t}
$$

so that

$$
\begin{aligned}
R_{2}(n, m, v, a, b, c, 0,0,0,0) & =2 \int_{0}^{\infty} f(t) t^{-m / 2} e^{-c t-q t-b / t} d t \int_{0}^{\infty} s^{m+v-3}\left(s^{2}+t\right)^{-\frac{m}{2}-\frac{n}{2}-v+2} \\
& \times \exp \left(-\frac{a-b}{s^{2}+t}\right) d s
\end{aligned}
$$

Changing variables to

$$
w=\frac{a-b}{s^{2}+t}
$$


transforms the s-integral to

$$
\frac{1}{a-b} \int_{0}^{\frac{a-b}{t}} e^{-w}\left(\frac{a-b}{w}\right)^{-\frac{m}{2}-\frac{n}{2}-v+4}\left(\frac{\sqrt{a-b-t w}}{\sqrt{w}}\right)^{m+v-4} d w .
$$

The last term in the integrand is turned into a square if $m=v=3$ and the middle term is flipped upside-down if $n \geq 1$, so letting $\{n=1, m=v=3\}$ gives $[8,9]$

$$
\begin{aligned}
& R_{2}(1,3,3, a, b, c, 0,0,0,0)=\quad \int_{0}^{\infty} \int_{0}^{\infty} \frac{1}{x^{1 / 2} y^{3 / 2}(x+y)^{3 / 2}} f\left(\frac{x y}{x+y}\right) e^{-\frac{a}{x}-\frac{b}{y}-c x y /(x+y)} d x d y \\
& =(a-b)^{-2} \int_{0}^{\infty} f(t) t^{-3 / 2} e^{-c t}\left(t e^{-a / t}-e^{-b / t}(-a+b+t)\right) d t \quad, \\
& \overrightarrow{f(t) \rightarrow t^{\mu}} \frac{2 c^{-\frac{\mu}{2}-\frac{1}{4}}\left(b^{1 / 4} a^{\frac{\mu}{2}+\frac{1}{4}} K_{-\mu-\frac{1}{2}}(2 \sqrt{a} \sqrt{c})-b^{\mu / 2}\left(\sqrt{c}(b-a) K_{\frac{1}{2}-\mu}(2 \sqrt{b} \sqrt{c})+\sqrt{b} K_{-\mu-\frac{1}{2}}(2 \sqrt{b} \sqrt{c})\right)\right)}{b^{1 / 4}(a-b)^{2}}
\end{aligned}
$$

where in the last line we have analytically integrated [7] the general form for the special case $f(t)=t^{\mu}$. This shows that the singularity at the origin, $y^{-3 / 2}$ and $t^{-3 / 2}$ in the two forms, does not preclude integration when one has a factor like $\exp ^{-a / t}$ to counterbalance it. Mathematica indeed does complain during numerical integration that the $d x d y$ integral "has evaluated to non-numerical values for all sampling points in the region with boundaries $\{\{\infty, 0\},\{\infty, 0\}\} "$ but nevertheless produces a value for $\mu=0$ for each integral, 9.384708 , that matches the analytical value 9.384708018702616 to seven places, for $\mathrm{a}=0.17, \mathrm{~b}=0.11$, and $\mathrm{c}=0.13$. One may even take $\mu<0$ with no problems.

Letting $\{n=m=v=3\}$ gives $[9,10]$

$$
\begin{aligned}
R_{2}(3,3,3, a, b, c, 0,0,0,0) & =\int_{0}^{\infty} \int_{0}^{\infty} \frac{1}{x^{3 / 2} y^{3 / 2}(x+y)^{3 / 2}} f\left(\frac{x y}{x+y}\right) e^{-\frac{a}{x}-\frac{b}{y}-c x y /(x+y)} d x d y \\
& =(a-b)^{-3} \int_{0}^{\infty} f(t) t^{-3 / 2} e^{-c t}\left(e^{-b / t}(a-b-2 t)+e^{-a / t}(a-b+2 t)\right) d t
\end{aligned}
$$

and so on. Numerical integration of both forms, with $f(t)=t^{3 / 2}$, gives $R_{2}(3,3,3,0.17,0.11,0.13,0,0,0,0)=1.117014$.

The limit of what one may integrate is $e^{-w} \sqrt{\frac{1}{w}}(a-b-t w)$ so that $\{n=0, m=v=3\}$ gives [11]

$$
\begin{aligned}
R_{2}(0,3,3, a, b, c, 0,0,0,0) & =\int_{0}^{\infty} \int_{0}^{\infty} \frac{1}{y^{3 / 2}(x+y)^{3 / 2}} f_{2}\left(\frac{x y}{x+y}\right) e^{-\frac{a}{x}-\frac{b}{y}-c x y /(x+y)} d x d y \\
& =(a-b)^{-3 / 2} \int_{0}^{\infty} f(t) t^{-3 / 2} e^{-c t} \\
& \times\left(\frac{1}{2} \sqrt{\pi}(2 a-2 b-t) e^{-b / t} \operatorname{erf}\left(\sqrt{\frac{a-b}{t}}\right)+\sqrt{t} \sqrt{a-b} e^{-a / t}\right) d t
\end{aligned}
$$

Numerical integration of both forms, with $f(t)=t^{3 / 2}$, gives $R_{2}(1,3,3,0.17,0.11,0.13,0,0,0,0)=$ 0.097224 , though Mathematica did better in the interval $1 / 2<\mu<3 / 2$ using adaptive quasi-Monte Carlo integration than the default method.

If instead we set the last term to unity with $m=v=2$ one gets for $\{n=1, m=v=2\}$ [12]

$$
\begin{aligned}
R_{2}(1,2,2, a, b, c, 0,0,0,0) & =\int_{0}^{\infty} \int_{0}^{\infty} \frac{1}{x^{1 / 2} y(x+y)} f\left(\frac{x y}{x+y}\right) e^{-\frac{a}{x}-\frac{b}{y}-c x y /(x+y)} d x d y \\
& =(a-b)^{-1 / 2} \int_{0}^{\infty} f(t) t^{-1} e^{-c t} e^{-b / t}\left(\sqrt{\pi} \operatorname{erf}\left(\sqrt{\frac{a-b}{t}}\right)\right) d t
\end{aligned}
$$

Numerical integration of both forms, with $f(t)=t^{3 / 2}$, gives $R_{2}(1,2,2,0.17,0.11,0.13,0,0,0,0)=$ 14.23627. For $\{n=m=v=2\}$ [8] 


$$
\begin{aligned}
R_{2}(1,2,2, a, b, c, 0,0,0,0) & =\int_{0}^{\infty} \int_{0}^{\infty} \frac{1}{x y(x+y)} f\left(\frac{x y}{x+y}\right) e^{-\frac{a}{x}-\frac{b}{y}-c x y /(x+y)} d x d y \\
& =(a-b)^{-1} \int_{0}^{\infty} f(t) t^{-3 / 2} e^{-c t}\left(e^{-b / t}-e^{-a / t}\right) d t
\end{aligned}
$$

Numerical integration of both forms, with $f(t)=t^{3 / 2}$, gives $R_{2}(1,2,2,0.17,0.11,0.13,0,0,0,0)=$ 3.755877. The extension to larger values of $n$ is straightforward.

Finally, we note that the results in this section are not well defined for $a=b$. Since the complicating exponential in the second line of (32) goes to unity in this case, we may write the general form

$$
\begin{aligned}
& R_{2}(n, m, v, b, b, c, 0,0,0,0)= \int_{0}^{\infty} \int_{0}^{\infty} \frac{1}{x^{n / 2} y^{m / 2}(x+y)^{v / 2}} f\left(\frac{x y}{x+y}\right) e^{-\frac{b}{x}-\frac{b}{y}-c x y /(x+y)} d x d y \\
&= \frac{\Gamma\left(\frac{1}{2}(m+v-2)\right) \Gamma\left(\frac{1}{2}(n+v-2)\right)}{\Gamma\left(\frac{1}{2}(m+n+2 v-4)\right)} \int_{0}^{\infty} f(t) e^{-c t} e^{-b / t} t^{\frac{1}{2}(-m-n-v+2)} d t . \\
& \Re(m+v)>2 \wedge \Re(n+v)>2
\end{aligned}
$$

This is most easily proved by setting $a=b$ and $h=0$ in Equation (60) of Section 5. The restrictions on $n, m$, and $v$ most obviously keep the gamma functions from becoming infinite when their arguments are integers less than one, but one may show by numerical integration that the above equation also does not hold for nonintegral values of $(m+v)<2$ even if the (erroneous) results are finite. Numerical integration of both forms, with $f(t)=t^{3 / 2}$, gives $R_{2}(2,3,1,0.11,0.11,0.13,0,0,0,0)=17.107195$.

\section{Set of Integral Reductions for More Complicated Exponentials}

A entirely different set of integral reductions may be crafted that include both positive and negative powers in the exponentials. Using the same partial fraction decomposition as in Equation (31) gives

$$
\begin{aligned}
R_{2}(n, m, v, a, b, c, 0,0, p, q) & =\int_{0}^{\infty} \int_{0}^{\infty} \frac{1}{y^{m / 2} x^{n / 2}(x+y)^{v / 2}} f\left(\frac{x y}{x+y}\right) e^{-\frac{a}{x}-\frac{b}{y}-c x y /(x+y)-p x-q y} d x d y \\
& =2 \int_{0}^{\infty} f(t) t^{-m / 2} e^{-c t-q t-b / t} d t \int_{0}^{\infty} s^{m+v-3}\left(s^{2}+t\right)^{-\frac{m}{2}-\frac{n}{2}-v+2} \\
& \times \exp \left(-\frac{a-b}{s^{2}+t}-p\left(s^{2}+t\right)-\frac{q t^{2}}{s^{2}}\right) d s
\end{aligned}
$$

and we may well have convergence problems for $b>a$ unless $c$ is large enough. Note that the exponential $e^{-c x y /(x+y)}$ multiplying $f\left(\frac{x y}{x+y}\right)$ is transformed as $e^{-c t}$ multiplying $f(t)$ as one would expect of a factor that can be folded into the definition of $f$.

If we then specialize this integral to the case where $q=0$, one may complete the square in the latter exponential and change variables

$$
\begin{aligned}
\exp \left(-\frac{a-b}{s^{2}+t}-p\left(s^{2}+t\right)\right) & =\exp \left(-\left(\sqrt{p} \sqrt{s^{2}+t}-\frac{\sqrt{a-b}}{\sqrt{s^{2}+t}}\right)^{2}-2 \sqrt{p} \sqrt{a-b}\right) \\
& \equiv \exp \left(-v^{2}-2 \sqrt{p} \sqrt{a-b}\right) .
\end{aligned}
$$


One normally examines each of the (in this case four) possible solutions in turn to see if any have properties that rule them out as unphysical. We start with

$$
s=-\sqrt{\frac{\sqrt{4 \sqrt{p} v^{2} \sqrt{a-b}+4 p(a-b)-4 a p+4 b p+v^{4}}}{2 p}+\frac{\sqrt{a-b}}{\sqrt{p}}+\frac{v^{2}}{2 p}-t .}
$$

Then with

$$
d s=\frac{\left(s^{2}+t\right)^{3 / 2}}{s\left(\sqrt{a-b}+\sqrt{p}\left(s^{2}+t\right)\right)} d v
$$

we have [6]

$$
\begin{aligned}
& R_{2}(n, m, v, a, b, c, 0,0, p, 0)=2 \int_{0}^{\infty} f(t) t^{-m / 2} e^{-c t-b / t-2 \sqrt{p} \sqrt{a-b}} d t \int_{\sqrt{p} \sqrt{t}-\sqrt{a-b} / \sqrt{t}}^{\infty} d v e^{-v^{2}} \\
& \times \frac{\sqrt{p} 2^{\frac{1}{2}(n+v-1)}\left(\frac{\sqrt{4 \sqrt{p} v^{2} \sqrt{a-b}+v^{4}}+2 \sqrt{p} \sqrt{a-b}+v^{2}}{p}\right)^{\frac{1}{2}(-m-n-2 v+7)}}{\left(\sqrt{4 \sqrt{p} v^{2} \sqrt{a-b}+v^{4}}+4 \sqrt{p} \sqrt{a-b}+v^{2}\right)}
\end{aligned}
$$

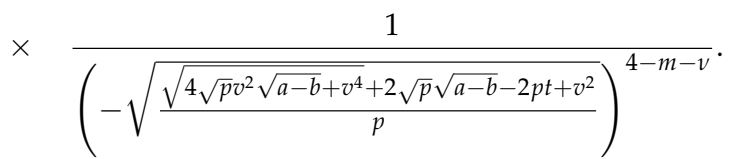

We set $\{m=4-v\}$, the last factor of (44) goes to unity, giving us some hope of doing the integral. However, even a further reduction to the simplest form, with $\{n=3-v\}$ [6]

$$
\begin{aligned}
& R_{2}(3-v, 4-v, v, a, b, c, 0,0, p, 0)=2 \int_{0}^{\infty} f(t) t^{-(4-v) / 2} e^{-c t-b / t-2 \sqrt{p} \sqrt{a-b}} d t \int_{\sqrt{p} \sqrt{t}-\sqrt{a-b} / \sqrt{t}}^{\infty} d v e^{-v^{2}} \\
& \times \frac{\sqrt{p} 2}{\left(\sqrt{4 \sqrt{p} v^{2} \sqrt{a-b}+v^{4}}+4 \sqrt{p} \sqrt{a-b}+v^{2}\right)} \\
& =2 \int_{0}^{\infty} f(t) t^{-m / 2} e^{-c t-b / t-2 \sqrt{p} \sqrt{a-b}} d t \\
& \times\left(\frac{t \sqrt{a-b} e^{4 \sqrt{p} \sqrt{a-b}} \sqrt{\frac{\left(-a+b+p t^{2}\right)^{2}}{t^{2}}} \operatorname{erf}\left(\frac{\sqrt{2 \sqrt{p} t \sqrt{a-b}+a-b+p t^{2}}}{\sqrt{t}}\right)}{\sqrt{2 \sqrt{p} t \sqrt{a-b}+a-b+p t^{2}}}\right. \\
& +(\sqrt{p} t \sqrt{a-b}-a+b) \operatorname{erf}\left(\frac{\sqrt{a-b}-\sqrt{p} t}{\sqrt{t}}\right) \\
& \left.+\left(e^{4 \sqrt{p} \sqrt{a-b}}-1\right)(-\sqrt{p} t \sqrt{a-b}+a-b)\right) \\
& \times \frac{\sqrt{\pi}}{8 \sqrt{p} \sqrt{a-b}(\sqrt{p} t \sqrt{a-b}-a+b)} \\
& {\left[\left(\frac{\sqrt{a-b}-\sqrt{p t}}{\sqrt{t}} \notin \mathbb{R} \vee \Re\left(\frac{\sqrt{a-b}-\sqrt{p} t}{\sqrt{t}}\right) \leq 0\right)\right.}
\end{aligned}
$$

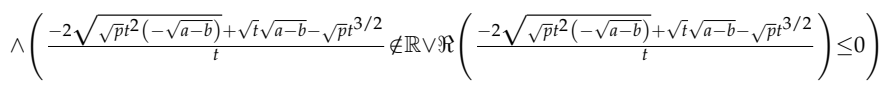

$$
\begin{aligned}
& \left.\wedge\left(\frac{2 \sqrt{\sqrt{p^{2}}(-\sqrt{a-b})}+\sqrt{t} \sqrt{a-b}-\sqrt{p p^{3} / 2}}{t} \notin \mathbb{R} \vee \Re\left(\frac{2 \sqrt{\left.\sqrt{p^{2}} t^{2}-\sqrt{a-b}\right)}+\sqrt{t} \sqrt{a-b}-\sqrt{p} \beta^{3 / 2}}{t}\right) \leq 0\right)\right]
\end{aligned}
$$

has conditions that cannot be met as $t$ becomes very small unless $\sqrt{p} t=\sqrt{a-b}$ or one of these coefficients in the original exponents is an imaginary number. Each of the other three solutions for $s(v)$ leads to similar difficulties with conditions that cannot be met. Numerical integration of $v$ also fails 
to give correct values for this reason, unless unless $\sqrt{a-b}$ is very close to zero for a wide variety of values for $n, m$, and $v$, even in the more general Equation (44).

Thus, let us return to the integral in terms of $x$ and $y$ to form a self-consistent version of the former condition by setting $p=(a-b)(x+y)^{2} /\left(x^{2} y^{2}\right)$. The utility of this value is not apparent in terms of the original variables (where $-\frac{a}{x}-\frac{b}{y}-p x \rightarrow \frac{x(b-a)}{y^{2}}+\frac{b-2 a}{x}+\frac{b-2 a}{y}$ ) but creates a useful exponential when expressed in terms of $s$ and $t$ on the last line of the modified integral

$$
\begin{aligned}
\tilde{R}_{2}(n, m, v, a, b, c, q) & =\int_{0}^{\infty} \int_{0}^{\infty} \frac{1}{y^{m / 2} x^{n / 2}(x+y)^{v / 2}} f\left(\frac{x y}{x+y}\right) \\
& \times e^{-a / x-b / y-c x y /(x+y)-(a-b)(x+y)^{2} /\left(x y^{2}\right)-q y} d x d y \\
& =2 \int_{0}^{\infty} f(t) t^{-m / 2} e^{-c t-q t-b / t} d t \int_{0}^{\infty} s^{m+v-3}\left(s^{2}+t\right)^{-\frac{m}{2}-\frac{n}{2}-v+2} \\
& \times \exp \left(-\frac{a-b}{s^{2}+t}-\frac{(a-b)\left(s^{2}+t\right)}{t^{2}}-\frac{q t^{2}}{s^{2}}\right) d s
\end{aligned}
$$

We again specialize this integral to the case where $q=0$ and complete the square in the latter exponential and change variables

$$
\begin{aligned}
\exp \left(-\frac{a-b}{s^{2}+t}-\frac{(a-b)\left(s^{2}+t\right)}{t^{2}}\right) & =\exp \left(-\left(\frac{\sqrt{a-b} \sqrt{s^{2}+t}}{t}-\frac{\sqrt{a-b}}{\sqrt{s^{2}+t}}\right)^{2}-\frac{2(a-b)}{t}\right) \\
& \equiv \exp \left(-v^{2}-\frac{2(a-b)}{t}\right) .
\end{aligned}
$$

Of the four possible solutions, we choose the one with

$$
s=-\sqrt{\frac{t^{3 / 2} v \sqrt{4 a-4 b+t v^{2}}}{2(a-b)}+\frac{t^{2} v^{2}}{2(a-b)}} .
$$

Then with

$$
d s=\frac{s \sqrt{a-b}\left(s^{2}+2 t\right)}{t\left(s^{2}+t\right)^{3 / 2}} d v
$$

we have [6]

$$
\begin{aligned}
\tilde{R}_{2}(n, m, v, a, b, c, p, 0) & =2 \int_{0}^{\infty} f(t) t^{-m / 2} e^{-c t-b / t-2(a-b) / t} d t \int_{0}^{\infty} d v e^{-v^{2}}\left(\frac{1}{a-b}\right)^{\frac{1}{2}(-n-v+2)} \\
\times & \frac{(-1)^{m+v-2} 2^{\frac{1}{2}(n+v-1)}\left(t\left(\sqrt{t} v \sqrt{4 a-4 b+t v^{2}}+2 a-2 b+t v^{2}\right)\right)^{\frac{1}{2}(-m-n-2 v+7)}}{\left(\sqrt{t} \sqrt{4 a-4 b+t v^{2}}+4 a-4 b+t v^{2}\right)\left(t^{3 / 2} v \sqrt{4 a-4 b+t v^{2}}+t^{2} v^{2}\right)^{\frac{1}{2}(4-m-v)}}
\end{aligned}
$$

If we set $\{m=4-v\}$ the second factor in the denominator of (50) goes to unity, as does the last factor in the numerator if we then set $\{n=3-v\}$, giving [6] 


$$
\begin{aligned}
\tilde{R}_{2}(3-v, 4-v, v, a, b, c, 0)= & \int_{0}^{\infty} \int_{0}^{\infty} \frac{1}{x^{(3-v) / 2} y^{(4-v) / 2}(x+y)^{v / 2}} f\left(\frac{x y}{x+y}\right) \\
\times & e^{-a / x-b / y-c x y /(x+y)-(a-b)(x+y)^{2} /\left(x y^{2}\right)} d x d y \\
= & 2 \int_{0}^{\infty} f(t) t^{-m / 2} e^{-c t-b / t-2(a-b) / t} \frac{\sqrt{\pi}}{4 \sqrt{a-b}}\left(1-e^{4(a-b) / t} \operatorname{erfc}\left(\frac{2 \sqrt{a-b}}{\sqrt{t}}\right)\right) d t . \\
& {\left[\sqrt{\frac{\Im(b)-\Im(a)}{\Im(t)}} \leq 0 \vee \Re(a) \Im(t)+\Im(b) \Re(t) \geq \Im(a) \Re(t)+\Re(b) \Im(t),\right.} \\
& \frac{\sqrt{\Im(b)-\Im(a)}}{\sqrt{I m(t)}} \geq 0 \vee \Re(a)+\frac{\Im(b) \Re(t)}{\Im(t)} \geq \frac{\Im(a) \Re(t)}{\Im(t)}+\Re(b), \\
& \left.\frac{\sqrt{b-a}}{s q r t t} \notin \mathbb{R} \vee\left(\frac{\sqrt{b-a}}{\sqrt{t}} \neq 0 \wedge \Re\left(\frac{\sqrt{b-a}}{\sqrt{t}}\right)=0\right)\right]
\end{aligned}
$$

Numerical integration of both forms, with $f(t)=t^{3 / 2}$, gives $\tilde{R}_{2}(0,1,3,0.17,0.11,0.13,0)=30.93045$. The powers of $v$ can be folded into a redefintion of $f$.

If we instead retain the last factor in the numerator with unit power by setting $\{n=1-v\}$, after reducing we obtain [6]

$$
\begin{aligned}
\tilde{R}_{2}(1-v, 4-v, v, a, b, c, 0)= & \int_{0}^{\infty} \int_{0}^{\infty} \frac{1}{x^{(1-v) / 2} y^{(4-v) / 2}(x+y)^{v / 2}} f\left(\frac{x y}{x+y}\right) \\
\times & e^{-a / x-b / y-c x y /(x+y)-(a-b)(x+y)^{2} /\left(x y^{2}\right)} d x d y \\
= & 2 \int_{0}^{\infty} f(t) \frac{\sqrt{\pi} t^{\frac{v}{2}-1}}{2 \sqrt{a-b}} e^{-2(a-b) / t-5 b / t-c t} \\
\times & \left(e^{4 a / t}+e^{4 b / t}-e^{4 a / t} \operatorname{erf}\left(\frac{2 \sqrt{a-b}}{\sqrt{t}}\right)\right) d t . \\
& \left.\vee\left(\left(\frac{\sqrt{\Im(b)-\Im(a)}}{\sqrt{\Im(t)}} \notin \mathbb{R} \vee \Re\left(\frac{\sqrt{\Im(b)-\Im(a)}}{\sqrt{\Im(t)}}\right)=0\right) \wedge\left(\sqrt{\frac{\Im(b)-\Im(a)}{\Im(t)}} \notin \mathbb{R} \vee \Re\left(\sqrt{\frac{\Im(b)-\Im(a)}{\Im(t)}}\right) \leq 0\right)\right)\right]
\end{aligned}
$$

Numerical integration of both forms, with $f(t)=t^{3 / 2}$, gives $\tilde{R}_{2}(-2,1,3,0.17,0.11,0.13,0)=6107.0659$.

One can also move the second factor in the denominator of (50) into the numerator with unit power by setting $\{m=6-v\}$. We then set $\{n=1-v\}$ to eliminate the other term in the numerator, and reduce the resultant quotient to obtain [6]

$$
\begin{aligned}
\tilde{R}_{2}(1-v, 6-v, v, a, b, c, 0)= & \int_{0}^{\infty} \int_{0}^{\infty} \frac{1}{x^{(1-v) / 2} y^{(6-v) / 2}(x+y)^{v / 2}} f\left(\frac{x y}{x+y}\right) \\
\times & e^{-a / x-b / y-c x y /(x+y)-(a-b)(x+y)^{2} /\left(x y^{2}\right)} d x d y \\
= & 2 \int_{0}^{\infty} f(t) t^{-2+v / 2} e^{-c t-b / t-2(a-b) / t} \frac{\sqrt{\pi}}{2 \sqrt{a-b}} \operatorname{erfc}\left(\frac{2 \sqrt{a-b}}{\sqrt{t}}\right) d t . \\
& {\left[\Re(a-b)>0, \quad \Re(a)+\frac{\Im(b) \Re(t)}{\Im(t)} \geq \frac{\Im(a) \Re(t)}{\Im(t)}+\Re(b) \vee\left(\sqrt{\frac{\Im(b)-\Im(a)}{\Im(t)}} \leq 0 \wedge \frac{\sqrt{\Im(b)-\Im(a)}}{\sqrt{\Im(t)}} \geq 0\right),\right.} \\
& \left.\frac{a-b}{t} \notin \mathbb{R} \vee \Re\left(\frac{a-b}{t}\right) \geq 0\right]
\end{aligned}
$$

Numerical integration of both forms, with $f(t)=t^{3 / 2}$, gives $\tilde{R}_{2}(-2,3,3,0.17,0.11,0.13,0)=354.25118$. Again, the powers of $v$ can be folded into a redefintion of $f$.

One may also move the second factor in the denominator of (50) into the numerator with a power of two by setting $\{m=8-v\}$. Again we eliminate the other term in the numerator with 
$\{n=1-v\}$, and reduce the resultant quotient somewhat. Mathematica 7 was unable to do this integral, but Mathematica 9 could, with

$$
\begin{aligned}
& \tilde{R}_{2}(1-v, 8-v, v, a, b, c, 0)=\int_{0}^{\infty} \int_{0}^{\infty} \frac{1}{x^{(1-v) / 2} y^{(8-v) / 2}(x+y)^{v / 2}} f\left(\frac{x y}{x+y}\right) \\
& \times \quad e^{-a / x-b / y-c x y /(x+y)-(a-b)(x+y)^{2} /\left(x y^{2}\right)} d x d y \\
& =2 \int_{0}^{\infty} f(t) t^{-2+v / 2} e^{-c t-b / t-2(a-b) / t} d t \frac{t^{\frac{v}{2}-3}}{8(a-b)^{2}} \\
& \times \quad\left(\sqrt{\pi} \sqrt{a-b} e^{-\frac{4 b}{t}}\left(-8 b e^{\frac{4 a}{t}}+t\left(e^{\frac{4 b}{t}}-e^{\frac{4 a}{t}}\right)+8 a e^{\frac{4 a}{t}}\right) .\right. \\
& \left.-e^{-\frac{4 b}{t}}\left(\sqrt{\pi} \sqrt{a-b} e^{\frac{4 a}{t}}(8 a-8 b-t) \operatorname{erf}\left(\frac{2 \sqrt{a-b}}{\sqrt{t}}\right)+4 \sqrt{t}(a-b) e^{\frac{4 b}{t}}\right)\right) \\
& {\left[\Re(a) \geq \Re(b), \Re(a)+\frac{\Im(b) \Re(t)}{\Im(t)} \geq \frac{\Im(a) \Re(t)}{\Im(t)}+\Re(b)\right.} \\
& \left.\vee\left(\left(\frac{\sqrt{\Im(b)-\Im(a)}}{\sqrt{\Im(t)}} \notin \mathbb{R} \vee \Re\left(\frac{\sqrt{\Im(b)-\Im(a)}}{\sqrt{\Im(t)}}\right)=0\right) \wedge\left(\sqrt{\frac{\Im(b)-\Im(a)}{\Im(t)}} \notin \mathbb{R} \vee \Re\left(\sqrt{\frac{\Im(b)-\Im(a)}{\Im(t)}}\right) \leq 0\right)\right)\right]
\end{aligned}
$$

Numerical integration of both forms, with $f(t)=t^{3 / 2}$, gives $\tilde{R}_{2}(-2,5,3,0.17,0.11,0.13,0)=0.5599498$.

Finally, one can also move the second factor in the denominator of (50) into the numerator with unit power by setting $\{m=6-v\}$ while allowing the other term in the numerator to appear with unit power by setting $\{n=-1-v\}$, and reduce the resultant quotient, giving

$$
\begin{aligned}
\tilde{R}_{2}(-1-v, 6-v, v, a, b, c, 0)= & \int_{0}^{\infty} \int_{0}^{\infty} \frac{1}{x^{(-1-v) / 2} y^{(6-v) / 2}(x+y)^{v / 2}} f\left(\frac{x y}{x+y}\right) \\
\times & e^{-a / x-b / y-c x y /(x+y)-(a-b)(x+y)^{2} /\left(x y^{2}\right)} d x d y \\
= & 2 \int_{0}^{\infty} f(t) t^{-1+v / 2} e^{-c t-b / t-2(a-b) / t} d t \\
\times & \left(e^{-\frac{4 b}{t}}\left(\sqrt{\pi} e^{\frac{4 a}{t}}(4 a-4 b-t) \operatorname{erf}\left(\frac{2 \sqrt{a-b}}{\sqrt{t}}\right)+4 \sqrt{t} \sqrt{a-b} e^{\frac{4 b}{t}}\right)\right. \\
+ & \left.\left(\sqrt{\pi} e^{-\frac{4 b}{t}}\left(4 b e^{\frac{4 a}{t}}+t\left(e^{\frac{4 a}{t}}+e^{\frac{4 b}{t}}\right)-4 a e^{\frac{4 a}{t}}\right)\right) \frac{1}{4(a-b)^{3 / 2}}\right) . \\
& \left.\left(\left(\frac{\sqrt{3(a) \geq \Re(b)}) \Re(a)+\frac{\Im(b) \Re(t)}{3(t)} \geq \frac{\Im(a) \Re(t)}{3(t)}+\Re(b) \vee}{\sqrt{3(t)}} \notin \mathbb{R} \vee \Re\left(\frac{\sqrt{3(b)-\Im(a)}}{\sqrt{\Im(t)}}\right)=0\right) \wedge\left(\sqrt{\frac{\Im(b)-\Im(a)}{\Im(t)}} n \text { otin } \mathbb{R} \vee \Re\left(\sqrt{\frac{\Im(b)-\Im(a)}{\Im(t)}}\right) \leq 0\right)\right)\right]
\end{aligned}
$$

Numerical integration of both forms, with $f(t)=t^{3 / 2}$, gives $\tilde{R}_{2}(-2,5,3,0.17,0.11,0.13,0)=1.254474 \times 10^{6}$.

\section{Set of Integral Reductions for Inverse Powers and Powers Times Inverse Binomials in The Exponentials}

\subsection{Integrals with $j=p=q=0$}

An entirely different set of integral reductions may be crafted for inverse powers in the exponentials. If we set $j=p=q=0$ in (7) we obtain

$$
\begin{aligned}
R_{2}(n, m, v, a, b, c, h, 0,0,0) & =\int_{0}^{\infty} \int_{0}^{\infty} \frac{1}{y^{m / 2} x^{n / 2}(x+y)^{v / 2}} f\left(\frac{x y}{x+y}\right) e^{-\frac{a}{x}-\frac{b}{y}-c x y /(x+y)-h y /(x+y)} d x d y \\
& =2 \int_{0}^{\infty} f(t) t^{-m / 2} e^{-c t-b / t} d t \int_{0}^{\infty} s^{m+v-3}\left(s^{2}+t\right)^{-\frac{m}{2}-\frac{n}{2}-v+2} \\
& \times \exp \left(-\frac{a-b+h t}{s^{2}+t}\right) d s
\end{aligned}
$$

If we change variables to

$$
w=\frac{a-b+h t}{s^{2}+t}, s=\frac{\sqrt{a-b+h t-t w}}{\sqrt{w}}
$$


with

$$
d s=-\frac{\left(s^{2}+t\right)^{2}}{2 s(a-b+h t)} d w
$$

we have

$$
\begin{aligned}
R_{2}(n, m, v, a, b, c, h, 0,0,0) & =\int_{0}^{\infty} \int_{0}^{\infty} \frac{1}{x^{n / 2} y^{m / 2}(x+y)^{v / 2}} f\left(\frac{x y}{x+y}\right) e^{-\frac{a}{x}-\frac{b}{y}-c x y /(x+y)-h y /(x+y)} d x d y \\
& =2 \int_{0}^{\infty} f(t) t^{-m / 2} e^{-c t-b / t}(a-b+h t)^{-m / 2-n / 2-v+3} d t \\
& \times \int_{0}^{(a-b+h t) / 2} w^{n / 2+v / 2-2} e^{-w}(a-b+h t-t w)^{m / 2+v / 2-2} d w
\end{aligned}
$$

The general result, good for $(m+v)>2$ and $(n+v)>2$ is

$$
\begin{aligned}
& R_{2}(n, m, v, a, b, c, h, 0,0,0)= \int_{0}^{\infty} \int_{0}^{\infty} \frac{1}{x^{n / 2} y^{m / 2}(x+y)^{v / 2}} f\left(\frac{x y}{x+y}\right) e^{-\frac{a}{x}-\frac{b}{y}-c x y /(x+y)-h y /(x+y)} d x d y \\
&= \int_{0}^{\infty} f(t) e^{-\frac{b}{t}-c t} t^{\frac{1}{2}(-m-n-v+2)} \frac{\Gamma\left(\frac{1}{2}(m+v-2)\right) \Gamma\left(\frac{1}{2}(n+v-2)\right)}{\Gamma\left(\frac{1}{2}(m+n+2 v-4)\right)} \\
& \times \quad{ }_{1} F_{1}\left(\frac{1}{2}(n+v-2) ; \frac{1}{2}(m+n+2 v-4) ;-\frac{a-b+h t}{t}\right) d t \\
& \\
& {[\Re(m+v)>2, \Re(n+v)>2,\{a, b, t, h\} \in \mathbb{R}, t>0,} \\
&((a=b \wedge h>0) \vee((a-b+t h)>0 \wedge b \neq a)]
\end{aligned}
$$

Numerical integration of all four forms, with $f(t)=t^{3 / 2}$, gives $R_{2}(3,3,0,0.17,0.11,0.13,0.23,0,0,0)=10.573548$. Whenever $m=n \pm 2 M$, where $M$ is a non-negative integer, we may simplify this with [13]

$$
\begin{aligned}
{ }_{1} F_{1}(A ; 2 A-M ; z) z & =\Gamma\left(A-M-\frac{1}{2}\right)\left(\frac{z}{4}\right)^{M-A+\frac{1}{2}} e^{z / 2} \\
\times & \sum_{k=0}^{M} \frac{\left((-1)^{k}(-M)_{k}(2 A-2 M-1)_{k}\right)\left(A+k-M-\frac{1}{2}\right) I_{A+k-M-\frac{1}{2}}\left(\frac{z}{2}\right)}{(2 A-M)_{k} k !}, \\
{ }_{1} F_{1}(A ; 2 A ; z) & =2^{2 A-1} e^{z / 2}(-z)^{\frac{1}{2}-A} \Gamma\left(A+\frac{1}{2}\right) I_{A-\frac{1}{2}}\left(-\frac{z}{2}\right),
\end{aligned}
$$

and

$$
\begin{aligned}
{ }_{1} F_{1}(A ; 2 A+M ; z) & =\Gamma\left(A-\frac{1}{2}\right)\left(\frac{z}{4}\right)^{-A+\frac{1}{2}} e^{z / 2} \\
& \times \sum_{k=0}^{M} \frac{\left((-M)_{k}(2 A-1)_{k}\right)\left(A+k-\frac{1}{2}\right) I_{A+k-\frac{1}{2}}\left(\frac{z}{2}\right)}{(2 A+M)_{k} k !},
\end{aligned}
$$

that can be readily integrated whenever $n+v=2(A+1)$ is an even integer. Even when $v$ alone is an even integer, and $M$ is a non-negative integer, we may nevertheless find an integrable form with

$$
{ }_{1} F_{1}(A ; A-M ; z)=\frac{(-1)^{M} e^{z} M ! L_{M}^{A-M-1}(-z)}{(1-A)_{M}} .
$$

\subsection{Application}

Probability amplitudes in atomic physics and variational wave functions for many-electron atoms involve products of Yukawa or (their special case) Coulomb potentials and hydrogenic orbitals that 
may be derived from Yukawa potentials via derivatives. Consider, then, the case of two Yukawa potentials centered on different positions

$$
S_{1}^{\eta_{1} 0 \eta_{2} 0}\left(0 ; 0, x_{2}\right)=\int d^{3} x_{1} \frac{e^{-\eta_{1} x_{1}}}{x_{1}} \frac{e^{-\eta_{2} x_{12}}}{x_{12}} \equiv \int d^{3} x_{1} \frac{e^{-\eta_{1} x_{1}}}{x_{1}} \frac{e^{-\eta_{2}\left|\mathbf{x}_{1}-\mathbf{x}_{2}\right|}}{\left|\mathbf{x}_{1}-\mathbf{x}_{2}\right|}
$$

The author has given [14] an analytically reduced form for multidimensional integrals over any number of such products in terms of Gaussian transforms, generally with one remaining integral for each atomic center in the original integrals. Such a reduction in the present case from three to two integral dimensions is in no way dramatic, but does provide a soluble example for the utility of the (further) reduction formulae of this section. One may write down the final form for the above integral using the notational formalism in that paper, but the reduction may instead be easily found by completing the square in the Gaussian transform

$$
\begin{aligned}
S_{1}^{\eta_{1} 0 \eta_{2} 0}\left(0 ; 0, x_{2}\right) & =\int d^{3} x_{1} \frac{1}{\sqrt{\pi}} \int_{0}^{\infty} d \rho_{1} \frac{e^{-x_{1}^{2} \rho_{1}} e^{-\eta_{1}^{2} / 4 / \rho_{1}}}{\rho_{1}^{1 / 2}} \frac{1}{\sqrt{\pi}} \int_{0}^{\infty} d \rho_{2} \frac{e^{-x_{12}^{2} \rho_{2}} e^{-\eta_{2}^{2} / 4 / \rho_{2}}}{\rho_{2}^{1 / 2}} \\
& =\frac{1}{\pi} \int d^{3} x_{1}^{\prime} \int_{0}^{\infty} d \rho_{1} \frac{e^{-\eta_{1}^{2} / 4 / \rho_{1}}}{\rho_{1}^{1 / 2}} \int_{0}^{\infty} d \rho_{2} \frac{e^{-\eta_{2}^{2} / 4 / \rho_{2}}}{\rho_{2}^{1 / 2}} \\
& \times \exp \left(-\left(\rho_{1}+\rho_{2}\right) x_{1}^{\prime 2}-\frac{x_{2}^{2} \rho_{1} \rho_{2}}{\rho_{1}+\rho_{2}}\right)
\end{aligned}
$$

where we have changed variables from $\mathbf{x}_{1}$ to $\mathbf{x}_{1}^{\prime}=\mathbf{x}_{1}-\frac{\rho_{2}}{\rho_{1}+\rho_{2}} \mathbf{x}_{2}$ with unit Jacobian. Then the spatial integral may be done [15]

$$
\begin{aligned}
\int e^{-\left(\rho_{1}+\rho_{2}\right) x_{1}^{\prime 2}} d^{3} x_{1}^{\prime}= & 4 \pi \int_{0}^{\infty} e^{-\left(\rho_{1}+\rho_{2}\right) x_{1}^{\prime 2}} x_{1}^{\prime 2} d x_{1}^{\prime}=\frac{4 \pi^{1+1 / 2}}{2^{2}\left(\rho_{1}+\rho_{2}\right)^{3 / 2}} . \\
& {\left[\rho_{1}+\rho_{2}>0\right] }
\end{aligned}
$$

What is left is

$$
\begin{aligned}
S_{1}^{\eta_{1} 0 \eta_{2} 0}\left(0 ; 0, x_{2}\right) & =\pi^{1 / 2} \int_{0}^{\infty} d \rho_{1} \frac{e^{-\eta_{1}^{2} / 4 / \rho_{1}}}{\rho_{1}^{1 / 2}} \int_{0}^{\infty} d \rho_{2} \frac{e^{-\eta_{2}^{2} / 4 / \rho_{2}}}{\rho_{2}^{1 / 2}} \\
& \times \quad \frac{1}{\left(\rho_{1}+\rho_{2}\right)^{3 / 2}} \exp \left(-\frac{x_{2}^{2} \rho_{1} \rho_{2}}{\rho_{1}+\rho_{2}}\right) \\
= & \pi^{1 / 2} \quad R_{2}\left(4,4,0, \eta_{1}^{2} / 4, \eta_{2}^{2} / 4, x_{2}^{2}, 0,0,0,0\right)
\end{aligned}
$$

where in the last line we have taken our function to be

$$
f(t)=t^{\mu}
$$

and in the present case have $\mu=3 / 2$. We could also have set $\mu=0$ with $n=m=1$ and $v=3$. Then using (62) we have

$$
\begin{aligned}
\pi^{1 / 2} R_{2}\left(4,4,0, \eta_{1}^{2} / 4, \eta_{2}^{2} / 4, x_{2}^{2}, 0,0,0\right)= & \pi^{1 / 2} \int_{0}^{\infty} d t\left(\frac{4 e^{-t x_{2}^{2}}}{\sqrt{t}\left(\eta_{2}^{2}-\eta_{1}^{2}\right)}\right)\left(e^{-\eta_{1}^{2} / 4 / t}-e^{-\eta_{2}^{2} / 4 / t}\right) \\
= & \frac{4 \pi\left(e^{-x_{2} \eta_{1}}-e^{-x_{2} \eta_{2}}\right)}{x_{2}\left(\eta_{2}^{2}-\eta_{1}^{2}\right)}, \\
& {\left[\Re\left(x_{2}^{2}\right) \geq 0, \Re\left(\eta_{1}^{2}\right) \geq 0, \Re\left(\eta_{2}^{2}\right) \geq 0\right] }
\end{aligned}
$$

which is indeed the correct result. 
Suppose instead of a Yukawa potential in $\eta_{1}$ we have a hydrogenic $1 s$ wave function that may be had by differentiation:

$$
\begin{aligned}
S_{1 s, 1}^{\eta_{1} \eta_{2} 0}\left(0 ; 0, x_{2}\right) & =\int d^{3} x_{1} u_{1 s}^{\eta_{1}}\left(x_{1}\right) \frac{e^{-\eta_{2} x_{12}}}{x_{12}} \\
& =\int d^{3} x_{1} \frac{\eta_{1}^{3 / 2} e^{-\eta_{1} x_{1}}}{\sqrt{\pi}} \frac{e^{-\eta_{2} x_{12}}}{x_{12}} \\
& =-\frac{\eta_{1}^{3 / 2}}{\sqrt{\pi}} \frac{\partial}{\partial \eta_{1}} \int d^{3} x_{1} \frac{e^{-\eta_{1} x_{1}}}{x_{1}} \frac{e^{-\eta_{2} x_{12}}}{x_{12}} \\
& =-\frac{\eta_{1}^{3 / 2}}{\sqrt{\pi}} \frac{\partial}{\partial \eta_{1}} S_{1}^{\eta_{1} 0 \eta_{2} 0}\left(0, ; 0, x_{2}\right) \\
& =-\frac{\eta_{1}^{3 / 2}}{\sqrt{\pi}} \frac{\partial}{\partial \eta_{1}} \frac{4 \pi\left(e^{-\eta_{2} x_{2}}-e^{-\eta_{1} x_{2}}\right)}{x_{2}\left(\eta_{1}^{2}-\eta_{2}^{2}\right)} \\
& =\frac{8 \sqrt{\pi} \eta_{1}^{5 / 2}}{\left(\eta_{1}^{2}-\eta_{2}^{2}\right)^{2}}\left(\frac{1}{x_{2}} e^{-\eta_{2} x_{2}}-\left(\frac{\eta_{1}^{2}-\eta_{2}^{2}}{2 \eta_{1}}+\frac{1}{x_{2}}\right) e^{-\eta_{1} x_{2}}\right)
\end{aligned}
$$

One may take the limit $\eta_{2} \rightarrow \eta_{1}$ to obtain Equation (49) from a previous paper [14] that used a very different integration method:

$$
S_{1 s, 1}^{\eta_{2} \eta_{2} 0}\left(0 ; 0, x_{2}\right)=\frac{\sqrt{\pi}\left(1+x_{2} \eta_{2}\right)}{\sqrt{\eta_{2}}} e^{-\eta_{2} x_{2}}
$$

\section{Set of Integral Reductions for Inverse Powers and Inverse Binomials in The Exponentials}

\subsection{The Transformation}

We may use arbitrary values of $a$ and $b$ in integrals of exponentials that contain both inverse powers and $-j /(x+y)$. We obtain

$$
\begin{aligned}
R_{2}(n, m, v, a, b, c, h, j, 0,0) & =\int_{0}^{\infty} \int_{0}^{\infty} \frac{1}{y^{m / 2} x^{n / 2}(x+y)^{v / 2}} f\left(\frac{x y}{x+y}\right) \\
& \times e^{-\frac{a}{x}-\frac{b}{y}-c x y /(x+y)-h y /(x+y)-j /(x+y)} d x d y \\
& =2 \int_{0}^{\infty} f(t) t^{-m / 2} e^{-c t} d t \int_{0}^{\infty} s^{m+v-3}\left(s^{2}+t\right)^{-\frac{m}{2}-\frac{n}{2}-v+2} \\
& \times \exp \left(-\frac{a t+b s^{2}}{t\left(s^{2}+t\right)}-\frac{h t}{s^{2}+t}-\frac{j s^{2}}{\left(s^{2}+t\right)^{2}}\right) d s
\end{aligned}
$$

If we again change variables to

$$
r=\frac{1}{s^{2}+t}, s=\frac{\sqrt{1-r t}}{\sqrt{r}},
$$

with

$$
d s=-\frac{2 s}{\left(s^{2}+t\right)^{2}} d r
$$


we have

$$
\begin{aligned}
R_{2}(n, m, v, a, b, c, h, j, 0,0) & =\int_{0}^{\infty} f(t) t^{-m / 2} e^{-\frac{b}{t}-c t} d t \int_{0}^{1 / t} r^{\frac{n}{2}+\frac{v}{2}-2}(1-r t)^{\frac{m}{2}+\frac{v}{2}-2} \\
& \left.\times \exp \left[+j r^{2} t-r(a-b+h t+j)\right]\right] d r .
\end{aligned}
$$

This is integrable for even values of $n+v \geq 4$ if we set the second factor in the $r$ integral to unity with $m=-v+4$. One may also do a binomial expansion of $(1-r t)$ for even values of $m+v>4$. This is done by completing the square in the exponential and setting

$$
\mathrm{r}^{\prime}=r-\frac{a-b+h t+j}{2 j t}
$$

with unit Jacobian.

\subsection{Application}

Fourier transforms of products of Yukawa or Coulomb potentials and hydrogenic orbitals are more difficult than (65). Consider, for instance, the case of the Fourier Transform of a product of two Yukawa potentials centered on different positions

$$
S_{1}^{\eta_{1} 0 \eta_{2} 0}\left(\mathbf{k} ; 0, \mathbf{x}_{2}\right)=\int d^{3} x_{1} \frac{e^{-\eta_{1} x_{1}}}{x_{1}} \frac{e^{-\eta_{2}\left|\mathbf{x}_{1}-\mathbf{x}_{2}\right|}}{\left|\mathbf{x}_{1}-\mathbf{x}_{2}\right|} e^{-i \mathbf{k} \cdot \mathbf{x}_{1}}
$$

The procedure is as in Section 5.2 above, but with two extra terms in the exponential after completing the square:

$$
\begin{aligned}
S_{1}^{\eta_{1} 0 \eta_{2} 0}\left(\mathbf{k} ; 0, \mathbf{x}_{2}\right) & =\int d^{3} x_{1} \frac{1}{\sqrt{\pi}} \int_{0}^{\infty} d \rho_{1} \frac{e^{-x_{1}^{2} \rho_{1}} e^{-\eta_{1}^{2} / 4 / \rho_{1}}}{\rho_{1}^{1 / 2}} \frac{1}{\sqrt{\pi}} \int_{0}^{\infty} d \rho_{2} \frac{e^{-x_{12}^{2} \rho_{2}} e^{-\eta_{2}^{2} / 4 / \rho_{2}}}{\rho_{2}^{1 / 2}} e^{-i \mathbf{k} \cdot \mathbf{x}_{1}} \\
& =\frac{1}{\pi} \int d^{3} x_{1}^{\prime} \int_{0}^{\infty} d \rho_{1} \frac{e^{-\eta_{1}^{2} / 4 / \rho_{1}}}{\rho_{1}^{1 / 2}} \int_{0}^{\infty} d \rho_{2} \frac{e^{-\eta_{2}^{2} / 4 / \rho_{2}}}{\rho_{2}^{1 / 2}} \\
& \times \exp \left(-\left(\rho_{1}+\rho_{2}\right) x_{1}^{\prime 2}-\frac{x_{2}^{2} \rho_{1} \rho_{2}}{\rho_{1}+\rho_{2}}-\frac{\rho_{2} i \mathrm{k} \cdot \mathbf{x}_{2}}{\rho_{1}+\rho_{2}}-\frac{\mathrm{k}^{2}}{4\left(\rho_{1}+\rho_{2}\right)}\right) \\
& =\pi^{1 / 2} \int_{0}^{\infty} d \rho_{1} \frac{e^{-\eta_{1}^{2} / 4 / \rho_{1}}}{\rho_{1}^{1 / 2}} \int_{0}^{\infty} d \rho_{2} \frac{e^{-\eta_{2}^{2} / 4 / \rho_{2}}}{\rho_{2}^{1 / 2}} \\
& \times \frac{1}{\left(\rho_{1}+\rho_{2}\right)^{3 / 2}} \exp \left(-\frac{x_{2}^{2} \rho_{1} \rho_{2}}{\rho_{1}+\rho_{2}}-\frac{\rho_{2} i \mathrm{k} \cdot \mathbf{x}_{2}}{\rho_{1}+\rho_{2}}-\frac{\mathrm{k}^{2}}{4\left(\rho_{1}+\rho_{2}\right)}\right) \\
& =\pi^{1 / 2} R_{2}\left(4,4,0, \eta_{1}^{2} / 4, \eta_{2}^{2} / 4, x_{2}^{2}, i \mathrm{k} \cdot \mathbf{x}_{2}, \mathrm{k}^{2} / 4,0,0\right)
\end{aligned}
$$

where in the last line we have taken our function to be

$$
f(t)=t^{\mu}
$$

and in the present case have $\mu=3 / 2$. We could also have set $\mu=0$ with $n=m=1$ and $v=3$. 
Then

$$
\begin{aligned}
S_{1}^{\eta_{1} 0 \eta_{1} 0}\left(\mathbf{k} ; 0, \mathbf{x}_{2}\right) & =\pi^{1 / 2} R_{2}\left(4,4,0, \eta_{1}^{2} / 4, \eta_{2}^{2} / 4, x_{2}^{2}, \mathrm{i} \mathrm{k} \cdot \mathbf{x}_{2}, \mathrm{k}^{2}, 0,0\right) \\
& =\pi^{1 / 2} \int t^{-1 / 2} e^{-\frac{\eta_{2}^{2}}{4 t}-x_{2}^{2} t} d t \\
& \times \int_{0}^{1 / t} \exp \left[\mathrm{k}^{2} r^{2} t+r\left(-i t k \cdot x_{2}-\frac{k^{2}}{4}-\frac{\eta_{1}^{2}}{4}+\frac{\eta_{2}^{2}}{4}\right)\right] d r \\
& =\pi^{1 / 2} \int_{0}^{\infty} d t e^{-\frac{\eta_{2}^{2}}{4 t}-x_{2}^{2} t} \frac{\sqrt{\pi}}{k t} \exp \left(-\frac{\left(i \mathrm{k} \cdot \mathbf{x}_{2} t+\frac{k^{2}}{4}+\frac{\eta_{1}^{2}}{4}-\frac{\eta_{2}^{2}}{4}\right)^{2}}{k^{2} t}\right) \\
& \times\left(\operatorname{erfi}\left(\frac{i \mathrm{k} \cdot \mathbf{x}_{2} t+\frac{k^{2}}{4}+\frac{\eta_{1}^{2}}{4}-\frac{\eta_{2}^{2}}{4}}{k \sqrt{t}}\right)-\operatorname{erfi}\left(\frac{i \mathrm{k} \cdot \mathbf{x}_{2} t-\frac{k^{2}}{4}+\frac{\eta_{1}^{2}}{4}-\frac{\eta_{2}^{2}}{4}}{k \sqrt{t}}\right)\right)
\end{aligned}
$$

As a check one can instead change variables to $\tau=\frac{\rho_{2}}{\rho_{1}+\rho_{2}}=\frac{y}{x+y}$ in (79) to give [7]

$$
\begin{aligned}
S_{1}^{\eta_{1} 0 \eta_{2} 0}\left(\mathbf{k} ; 0, \mathbf{x}_{2}\right) & =\pi^{1 / 2} \int_{0}^{1} d \tau \frac{1}{\tau^{1 / 2}} e^{-i \mathbf{k} \cdot \mathbf{x}_{2} \tau} \int_{0}^{\infty} d x \frac{1}{x^{3 / 2}} \\
& \times \exp \left(-x_{2}^{2} \tau x-\frac{(1-\tau)\left(k^{2} \tau+\eta_{2}^{2}\right)+\eta_{1}^{2} \tau}{4 \tau x}\right) \\
& =\pi^{1 / 2} \int_{0}^{1} d \tau e^{-i \mathbf{k} \cdot \mathbf{x}_{2} \tau} \frac{2 \sqrt{\pi} \exp \left(-x_{2} \sqrt{(1-\tau)\left(k^{2} \tau+\eta_{2}^{2}\right)+\eta_{1}^{2} \tau}\right)}{\sqrt{(1-\tau)\left(k^{2} \tau+\eta_{2}^{2}\right)+\eta_{1}^{2} \tau}}, \\
& =2 \pi \int_{0}^{1} d \tau e^{-i \mathbf{k} \cdot \mathbf{x}_{2} \tau} \frac{\exp \left(-x_{2} L\right)}{L}
\end{aligned}
$$

where

$$
L=\sqrt{(1-\tau)\left(k^{2} \tau+\eta_{2}^{2}\right)+\eta_{1}^{2} \tau} .
$$

One can show numerically that these two integrals are equal, with $\pi^{1 / 2} R_{2}\left(4,4,0,(0.82)^{2} / 4,(0.66)^{2} / 4,(0.36)^{2}, \mathrm{i}(0.36)(0.19),(0.19)^{2}, 0,0\right)=6.4564-0.2108 \mathrm{i}$. Cheshire [16] reduced the related integral (his Equation (19))

$$
\begin{aligned}
I_{1} & =\left.\frac{\eta_{1}^{3 / 2}}{\sqrt{\pi}} S_{1 s 1}^{\eta_{1} 0 \eta_{2} 0}\left(\frac{1}{2} \mathbf{k}_{\mathbf{f}} ; 0, x_{2}\right)\right|_{\eta_{1}=1, \eta_{2}=1 / 2} \\
& =\left.\int d^{3} x_{1} \frac{\eta_{2}^{3 / 2}}{\sqrt{\pi}} e^{-\eta_{2} x_{12}} \frac{\eta_{1}^{3 / 2}}{\sqrt{\pi}} \frac{e^{-\eta_{1} x_{1}}}{x_{1}} e^{-i \frac{1}{2} \mathbf{k}_{\mathbf{f}} \cdot \mathbf{x}_{1}}\right|_{\eta_{1}=1, \eta_{2}=1 / 2} \\
& =\left.\frac{\eta_{1}^{3 / 2}}{\sqrt{\pi}} \frac{\eta_{2}^{3 / 2}}{\sqrt{\pi}}\left(-\frac{\partial}{\partial \eta_{2}}\right) \int d^{3} x_{1} \frac{e^{-\eta_{2} x_{12}}}{x_{12}} \frac{e^{-\eta_{1} x_{1}}}{x_{1}} e^{-i \frac{1}{2} \mathbf{k}_{\mathbf{f}} \cdot \mathbf{x}_{1}}\right|_{\eta_{1}=1, \eta_{2}=1 / 2} \\
& =\left.\frac{\eta_{1}^{3 / 2}}{\sqrt{\pi}} \frac{\eta_{2}^{3 / 2}}{\sqrt{\pi}}\left(-\frac{\partial}{\partial \eta_{2}}\right) S_{1}^{\eta_{1} 0 \eta_{2} 0}\left(\frac{1}{2} \mathbf{k}_{\mathbf{f}} ; 0, x_{2}\right)\right|_{\eta_{1}=1, \eta_{2}=1 / 2},
\end{aligned}
$$

which matches the present result after substituting the specialized values for $\eta_{1}$ and $\eta_{2}$.

If $\eta_{1}=\eta_{2}$ we have

$$
L=\sqrt{k^{2}(1-\tau) \tau+\eta_{1}^{2}}
$$


In the limit $k \rightarrow 0$ and if $\eta_{2}=\eta_{1}$ we can analytically integrate either form to give

$$
S_{1}^{\eta_{1} 0 \eta_{1} 0}\left(0 ; 0, x_{2}\right)=\frac{2 \pi e^{-x_{2} \eta_{1}}}{\eta_{1}} .
$$

\section{Conclusions}

We have found the means to reduce two-dimensional integrals that include exponentials, powers, and binomials along with a general function that depends on the integration variables in the specific configuration $f\left(\frac{x y}{x+y}\right)$ to a one-dimensional integral involving that same function of the new integration variable $f(t)$. The various solutions are grouped according to which terms are nonzero in the exponential $e^{-\frac{a}{x}-\frac{b}{y}-c x y /(x+y)-h y /(x+y)-j /(x+y)-p x-q y}$, and next by powers of the integration variables. Such integrals arise in Gaussian transforms of atomic, molecular, and optical transition amplitudes [2,3] and may appear in the calculation of expectation operators in percolation theory [4]. Two applications of the results for atomic transition amplitudes are given. The extension to three-dimensional integrals is worth pursuing since it would allow for the solution of many more problems in AMO theory.

Funding: This research received no external funding.

Acknowledgments: I would like to thank Ray A. Mayer, of Reed College, for creating a very different proof of (2) for me, and to his colleague Nicholas Wheeler for the introduction.

Conflicts of Interest: The author declares that there is no conflict of interest.

\section{References}

1. Prudnikov, A.P.; Brychkov, Y.A.; Marichev, O.I. Integrals and Series; Gordon and Breach: New York, NY, USA, 1986; Volume 1, p. 567.

2. Jack, S.C. Fourier transform of the multicenter product of 1s hydrogenic orbitals and Coulomb or Yukawa potentials and the analytically reduced form for subsequent integrals that include plane waves. Phys. Rev. A 1989, 39, 5062-5070. [CrossRef]

3. Straton, J.C. Reduced form for the general-state multicenter integral from an integro-differential transform. Phys. Rev. A 1990, 41, 71-77. [CrossRef] [PubMed]

4. Shang, Y. Multi-Type Directed Scale-Free Percolation. Commun. Theor. Phys. 2012, 57, 701-716. [CrossRef]

5. Gradshteyn, I.S.; Ryzhik, I.M. Table of Integrals, Series, and Products, 5th ed.; Academic: New York, NY, USA, 1994; p. 385.

6. Wolfram Mathematica. Available online: https://www.wolfram.com/mathematica/?source=nav (accessed on 30 August 2020.)

7. Gradshteyn, I.S.; Ryzhik, I.M. Table of Integrals, Series, and Products, 5th ed.; No. 8.432.8; Academic: New York, NY, USA, 1994; p. 969.

8. Gradshteyn, I.S.; Ryzhik, I.M. Table of Integrals, Series, and Products, 5th ed.; No. 2.311; Academic: New York, NY, USA, 1994; p. 111.

9. Gradshteyn, I.S.; Ryzhik, I.M. Table of Integrals, Series, and Products, 5th ed.; No. 2.322.1; Academic: New York, NY, USA, 1994; p. 112.

10. Gradshteyn, I.S.; Ryzhik, I.M. Table of Integrals, Series, and Products, 5th ed.; No. 2.322.2; Academic: New York, NY, USA, 1994; p. 112.

11. Gradshteyn, I.S.; Ryzhik, I.M. Table of Integrals, Series, and Products, 5th ed.; No. 3.381.1; Academic: New York, NY, USA, 1994; p. 364.

12. Gradshteyn, I.S.; Ryzhik, I.M. Table of Integrals, Series, and Products, 5th ed.; No. 3.361.1; Academic: New York, NY, USA, 1994; p. 362.

13. Prudnikov, A.P.; Brychkov, Y.A.; Marichev, O.I. Integrals and Series; No. 7.11.5-7; Gordon and Breach: New York, NY, USA, 1986; Volume 3; p. 579.

14. Straton, J.C. Analytically reduced form of multicenter integrals from Gaussian transforms. Phys. Rev. A 1989, 39, 1676-1684. [CrossRef] [PubMed] 
15. Gradshteyn, I.S.; Ryzhik, I.M. Table of Integrals, Series, and Products, 5th ed.; No. 3.461.2; Academic: New York, NY, USA, 1994; p. 382.

16. Cheshire, I.M. Positronium formation by fast positrons in atomic hydrogen. Proc. Phys. Soc. 1964, 83, $227-237$. [CrossRef]

(c) (

(C) 2020 by the author. Licensee MDPI, Basel, Switzerland. This article is an open access article distributed under the terms and conditions of the Creative Commons Attribution (CC BY) license (http:/ / creativecommons.org/licenses/by/4.0/). 